\title{
Selective Disruption of Nucleus Accumbens Gating Mechanisms in Rats Behaviorally Sensitized to Methamphetamine
}

\author{
Anne Marie Brady, Stanley D. Glick, and Patricio 0'Donnell \\ Center for Neuropharmacology and Neuroscience, Albany Medical College, Albany, New York 12208
}

\begin{abstract}
Behavioral sensitization of psychostimulant-induced locomotor activity in rats has been proposed as a model of addiction and is accompanied by neuroadaptations in the nucleus accumbens and related circuits. Here, we used in vivo intracellular recordings to examine electrophysiological properties of accumbens neurons from animals that did or did not exhibit behavioral sensitization after repeated methamphetamine $(5.0 \mathrm{mg} / \mathrm{kg} ; 5 \mathrm{~d})$. Although spontaneous activity of accumbens neurons was virtually unchanged, multiple synaptic interactions controlling membrane potential states were disrupted in sensitized animals. For example, stimulation of the ventral tegmental area attenuated accumbens responses to prefrontal cortex activation in nonsensitized and saline-treated animals, but not in sensitized animals. Acute methamphetamine $(0.5 \mathrm{mg} / \mathrm{kg})$ abolished accumbens up and down states in nonsensitized and saline-treated animals, suggesting a disruption of normal information processing in this area. However, acute methamphetamine failed to affect this pattern in accumbens neurons from sensitized animals. These results suggest that both acute and repeated methamphetamine administration can disrupt synaptic interactions in the nucleus accumbens; however, the nature of these alterations depends critically on the extent of behavioral sensitization. It is speculated that the response to acute methamphetamine in nonsensitized and saline-treated animals may be functionally adaptive, whereas the neuroadaptations observed in sensitized animals may be maladaptive and detrimental to accumbens information processing.
\end{abstract}

Key words: dopamine; glutamate; nucleus accumbens; addiction; behavioral sensitization; methamphetamine

\section{Introduction}

Behavioral sensitization in animals is characterized by an enhanced locomotor response to a psychostimulant after repeated experience with that drug. Sensitized locomotor activity or stereotypy has been observed after chronic treatment with amphetamine (Wolf et al., 1993; Paulson and Robinson, 1995; Mendrek et al., 1998), methamphetamine (Meth) (Higashi et al., 1989; Szumlinski et al., 2000), or cocaine (Pierce et al., 1998; Beurrier and Malenka, 2002), and may persist for several months (Paulson et al., 1991). Behavioral sensitization is thought to model aspects of addictive behavior, such as drug craving (Robinson and Berridge, 1993). Sensitized animals are faster to acquire drug selfadministration behavior (Deminiere et al., 1989) and reach higher break points on a progressive ratio schedule (Mendrek et al., 1998; Lorrain et al., 2000), and animals with a history of drug self-administration also exhibit sensitized locomotor activity (De

Received Feb. 17, 2005; revised June 7, 2005; accepted June 9, 2005

This work was supported by National Institutes of Health Grants DA14020 (P.0.) and DA07307 (S.D.G.) and National Research Service Award DA14821 (A.M.B.). We thank Nicole Barnhardt, Atara Marzouk, and Katrina Emmerich for their assistance with behavioral testing and histological processing and Brian Lowry for developing and maintaining the data acquisition software (Neuroscope)

Correspondence should be addressed to Dr. Patricio 0'Donnell, Albany Medical College (MC-136), $47 \mathrm{New}$ Scotland Avenue, Albany, NY 12208. E-mail: odonnep@mail.amc.edu.

A. M. Brady's present address: Department of Psychology, St. Mary's College of Maryland, St. Mary's City, MD 20686.

DOI:10.1523/JNEUROSCI.0643-05.2005

Copyright $\odot 2005$ Society for Neuroscience $\quad$ 0270-6474/05/256687-09\$15.00/0
Vries et al., 1998). Thus, although behavioral sensitization in rats is manifested by changes in locomotion, it may reflect neurobiological changes related to drug addiction.

The neural circuit involved in behavioral sensitization includes the prefrontal cortex (PFC), ventral tegmental area (VTA), and nucleus accumbens (Cador et al., 1995; Pierce and Kalivas, 1997). Activation of the glutamatergic pathway from the PFC to the VTA is necessary for the initiation of behavioral sensitization (Wolf et al., 1995; Cador et al., 1999). In contrast, the long-term expression of behavioral sensitization is accompanied by structural and functional neuroadaptations in the nucleus accumbens (Wolf et al., 1993; Paulson and Robinson, 1995; Robinson and Kolb, 2004), including alterations in dopaminergic and glutamatergic inputs to accumbens neurons (Beurrier and Malenka, 2002; Li and Kauer, 2004). Such changes may underlie inappropriate neural plasticity and learning that could ultimately lead to compulsive drug-seeking behavior (Kauer, 2004; Wolf et al., 2004). Thus, the repeated use of addictive drugs has been described as usurping neural mechanisms that underlie normal learning and motivational processes (Hyman et al., 2001).

The functional nature of drug-induced neuroadaptations in the nucleus accumbens remains unclear (Robinson and Kolb, 2004). Several in vitro electrophysiological studies have shown that excitatory drive onto accumbens neurons is reduced after repeated psychostimulant administration (White et al., 1995; Zhang et al., 1998; Thomas et al., 2001; Beurrier and Malenka, 
2002). However, the activity of accumbens neurons in vivo is fundamentally different and characterized by oscillations between a resting down state and a more depolarized, synaptically driven up state during which neurons may fire action potentials (O'Donnell and Grace, 1995; Goto and O'Donnell, 2001; Brady and O'Donnell, 2004), and which is not observed in vitro (O’Donnell and Grace, 1996). Furthermore, synaptic responses to afferent stimulation are different depending on the membrane potential state of accumbens neurons (O'Donnell and Grace, 1995; Goto and O'Donnell, 2002; Brady and O'Donnell, 2004), suggesting that these fluctuations play an important role in shaping information processing in the nucleus accumbens by providing a gating mechanism (O’Donnell, 1999). We sought to characterize the electrophysiological properties of accumbens neurons in vivo after repeated administration of Meth, with particular attention to individual differences among animals that did or did not exhibit behavioral sensitization.

\section{Materials and Methods}

\section{Behavioral protocol}

Male adult Sprague Dawley rats were obtained at a weight of 181-200 g (approximate age, $49 \mathrm{~d}$ ) from Taconic Farms (Germantown, NY) $(n=7)$ or Charles River Laboratories (Wilmington, MA) $(n=43)$. Animals were singly housed on arrival in a colony room with a $12 \mathrm{~h}$ light/dark cycle (lights on at 6:00 A.M.) and were allowed ad libitum access to food and water throughout the experiment. After 1 week of acclimation to the colony room, animals were briefly handled for $2 \mathrm{~d}$ before beginning behavioral testing. These experiments were conducted in accordance with the United States Public Health Service Guide for the Use and Care of Animals, and all procedures were approved by the Albany Medical College Institutional Animal Care and Use Committee.

On the first day of behavioral testing (baseline phase), animals were assessed for their locomotor activity in response to a challenge dose of Meth (0.5 mg/kg, i.p.; Sigma-Aldrich, St. Louis, MO) or saline. Locomotor activity was recorded in opaque, cylindrical activity chambers $(60 \mathrm{~cm}$ diameter; Med Associates, St. Albans, VT) with metal grid floors. An activity count was recorded whenever the animal interrupted one of three intersecting light beams, emitted from photocells equidistantly around the circumference of the chamber walls $3 \mathrm{~cm}$ above the floor. Activity counts were summated and recorded every $10 \mathrm{~min}$ on a Pentium computer using Med Associates software (Med-PC for Windows). To separate the effects of novelty from the pharmacological effects of Meth, all animals were initially given an injection of saline $(1 \mathrm{ml} / \mathrm{kg}$, i.p. $)$ and locomotor activity was recorded for $30 \mathrm{~min}$. After this habituation period, animals were briefly removed from the chambers and injected with Meth $(0.5 \mathrm{mg} / \mathrm{kg}$, i.p. $)$ or saline. Animals were then placed back in the chambers, and activity counts were recorded for an additional $2 \mathrm{~h}$.

Three days after the baseline locomotor activity procedure, daily injections (chronic phase) were begun. Animals that had received Meth during the baseline phase received five consecutive daily injections of Meth $(5.0 \mathrm{mg} / \mathrm{kg}$, i.p.), whereas animals that had received saline at baseline received five daily injections of saline $(1 \mathrm{ml} / \mathrm{kg}$, i.p.). All chronic injections were administered to animals in their home cages in the colony room. After the chronic phase, animals were left undisturbed (except for husbandry) for a period of $13 \mathrm{~d}$ (withdrawal phase). On the 14th day after the last chronic injection (test phase), animals were again tested for locomotor activity in response to the same challenge dose of Meth $(0.5$ $\mathrm{mg} / \mathrm{kg}$, i.p.) or saline used in the baseline phase. Activity counts were again recorded for $30 \mathrm{~min}$ after an injection of saline and for $2 \mathrm{~h}$ after injection of Meth or saline. For animals injected with Meth, the locomotor response to Meth during the test phase was compared with the response to Meth during the baseline phase, and a measure of sensitization (percent change from baseline, total activity counts) was computed for each individual animal. Based on this measure, animals were classified post hoc as sensitized ( $\geq 50 \%$ change from baseline) or nonsensitized ( $\leq 25 \%$ change from baseline). Animals exhibiting a change between 25 and $50 \%$ were not classified and were not used for subsequent electrophysiological recording.

\section{Electrophysiological recordings}

In vivo intracellular recordings were performed 3-11 d after the test phase. Animals were initially anesthetized with chloral hydrate $(400 \mathrm{mg} /$ kg, i.p.), and subsequent anesthesia was maintained via constant infusion of chloral hydrate $\left(20-30 \mathrm{mg} \cdot \mathrm{kg}^{-1} \cdot \mathrm{h}^{-1}\right.$, i.p.) using a syringe pump (Bioanalytical Systems, West Lafayette, IN). Animals were placed in a stereotaxic apparatus (David Kopf, Tujunga, CA), and bipolar concentric stimulating electrodes $[1 \mathrm{~mm}$ outer diameter (o.d.); $0.5 \mathrm{~mm}$ tip separation; Rhodes Medical Instruments, Woodland Hills, CA] were placed in the right medial PFC (from bregma, $3.0 \mathrm{~mm}$ rostral, $1.6 \mathrm{~mm}$ lateral, $4.3 \mathrm{~mm}$ ventral from skull; $30^{\circ}$ angle toward midline) and VTA ( $6.0 \mathrm{~mm}$ caudal, $0.5 \mathrm{~mm}$ lateral, $7.4 \mathrm{~mm}$ ventral from dura). Each stimulating electrode was connected to an Isoflex stimulus isolation unit (AMPI, Jerusalem, Israel), driven by a Master-8 stimulator (AMPI). Intracellular recording electrodes were pulled from 1-mm-o.d. borosilicate glass tubing (World Precision Instruments, Sarasota, FL) to a resistance of $\sim 40-100 \mathrm{M} \Omega$ (measured in situ) with a P-97 Flaming-Brown microelectrode puller (Sutter Instruments, Novato, CA). Recording electrodes were filled with $2 \%$ Neurobiotin (Vector Laboratories, Burlingame, CA) in $2 \mathrm{M}$ potassium acetate and lowered into the right nucleus accumbens with the aid of a hydraulic microdrive (Trent Wells, Coulterville, CA), within the following range of coordinates: $1.2-1.8 \mathrm{~mm}$ rostral, $1.2-1.7$ $\mathrm{mm}$ lateral, $5.5-7.5 \mathrm{~mm}$ ventral from cortical surface. Intracellular signals from the recording electrode were transmitted through a headstage connected to an intracellular amplifier (IR-283; Neurodata, Delaware Water Gap, PA), and the recording signal was continuously monitored on a digital oscilloscope (TDS-220; Tektronix, Beaverton, OR). Membrane potential recordings were acquired and digitized at $10-20$ $\mathrm{kHz}$ via an interface board (DAP-3215; Microstar Labs, Bellevue, WA) installed in a Pentium III computer using custom-designed software (Neuroscope)

After stable impalement of a neuron in the nucleus accumbens, at least 5 min of baseline recording were collected. A series of positive and negative current injections $(0.1-0.5 \mathrm{nA} ; 100 \mathrm{~ms})$ was then administered to determine the input resistance and time constant of the cell $(\tau)$ (time to reach $63 \%$ of maximal voltage response to a negative current step). The criteria used to identify recordings for additional data analysis included the following: (1) at least $5 \mathrm{~min}$ of stable baseline recording, (2) membrane potential of at least $-50 \mathrm{mV},(3)$ action potential amplitude of at least $40 \mathrm{mV}$ from threshold, and (4) $\tau$ value between 3 and $10 \mathrm{~ms}$. After baseline recordings, synaptic responses to PFC and VTA stimulation were investigated. The PFC was stimulated with a single pulse $(0.8-1.0$ $\mathrm{mA} ; 0.5 \mathrm{~ms}$ ), whereas stimulation of the VTA consisted of a $20 \mathrm{~Hz}$ train of five pulses ( $1.0 \mathrm{~mA} ; 0.5 \mathrm{~ms}$ ). The ability of VTA stimulation to attenuate the response to PFC stimulation (Brady and O'Donnell, 2004) was also investigated with a protocol in which PFC pulses were delivered $900 \mathrm{~ms}$ before and $200 \mathrm{~ms}$ after train stimulation of the VTA. All stimulations were delivered once every $15 \mathrm{~s}$ for $10-15$ repetitions, and responses to stimulation were averaged over all repetitions delivered to the cell. Baseline activity in some cells was also recorded after acute administration of Meth, at the same challenge dose used in the baseline and test phases of the behavioral protocol $(0.5 \mathrm{mg} / \mathrm{kg}$, i.p.).

\section{Histology}

After the completion of recording, Neurobiotin was injected into the cell by passing positive current $(0.5-1.0 \mathrm{nA} ; 200 \mathrm{~ms}$ pulses; $2 \mathrm{~Hz})$ for $20-40$ min through the recording electrode. At the completion of the experiment, animals were killed with an overdose of pentobarbital $(100 \mathrm{mg} / \mathrm{kg})$ and transcardially perfused with cold saline followed by cold $4 \%$ paraformaldehyde. Brains were postfixed in $4 \%$ paraformaldehyde for at least $24 \mathrm{~h}$ before being transferred to a solution of $30 \%$ sucrose in $0.1 \mathrm{M}$ phosphate buffer for cryoprotection. Sections were cut $(30-40 \mu \mathrm{m})$ using a freezing microtome and placed in phosphate buffer. Sections through the PFC and VTA were mounted on gelatin-coated slides and Nissl stained to verify placement of stimulating electrodes. Sections through the nucleus accumbens were processed for visualization of Neurobiotin-filled cells and were counterstained for calbindin immunoreactivity (anti-calbindin D-28K; Vector Laboratories; 1:30,000) to distinguish cell locations in the core and shell regions of the accumbens. 
a

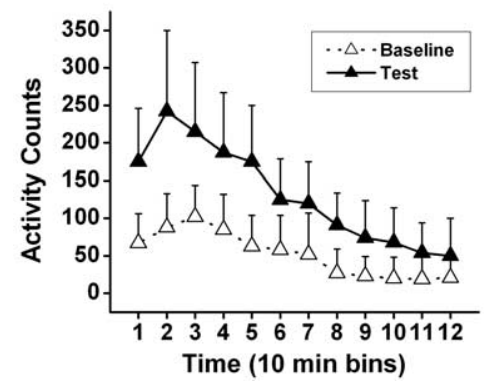

C

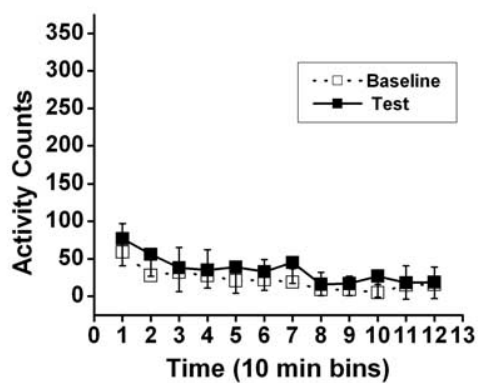

b

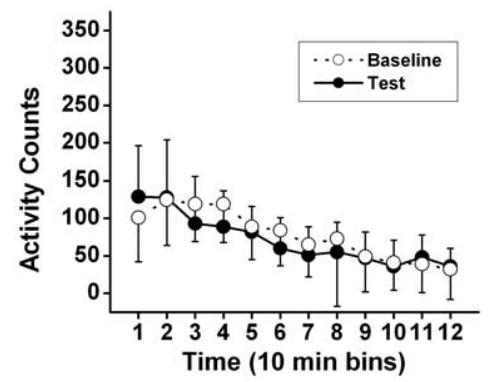

d

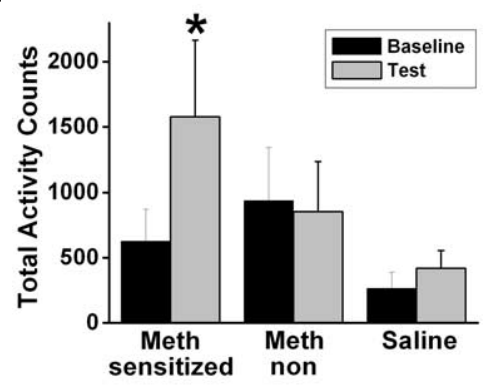

Figure 1. Locomotor activity (mean \pm SD) in animals after repeated injections of Meth or saline. Only animals from which successful intracellular recordings were subsequently obtained are included here. $\boldsymbol{a}$, Animals classified as behaviorally sensitized ( $n=22$ ) exhibited a stronger and longer-lasting locomotor response to Meth during the test phase compared with the baseline phase. $\boldsymbol{b}$, Animals classified as nonsensitized $(n=13)$ exhibited no significant changes in locomotor activity in response to Meth injection from the baseline to the test phase. $c$, Saline-treated animals $(n=15)$ exhibited a low level of locomotor activity in response to saline injection during both phases. $\boldsymbol{d}$, Total activity counts (collapsed across the $2 \mathrm{~h}$ behavior period) for each of the three groups. ${ }^{*} p<0.001$ versus baseline. Error bars indicate SD.

\section{Statistical analysis}

Behavioral data. Locomotor activity counts during the $30 \mathrm{~min}$ habituation period (after saline injection in all animals) were analyzed with a mixed ANOVA for effects of session (baseline vs test phase), time (3 bins), and group. Locomotor activity counts during the $2 \mathrm{~h}$ activity period (after Meth or saline injection) were analyzed for effects of session and time (12 bins) using separate repeated-measures ANOVAs on data from sensitized, nonsensitized, and saline-treated animals. Significant interactions were followed up with one-way ANOVAs or $t$ tests, and significant main effects were explored using dependent $t$ tests. An $\alpha$ level of 0.05 was used for all analyses.

Electrophysiological data. Multiple baseline dependent measures (membrane potential, up-state duration, up-state frequency, percentage of time in up state, spike threshold, spike amplitude, spike duration, firing rate, input resistance, time constant, and rheobase) were analyzed using a series of randomized ANOVAs. First, data from all recorded cells were analyzed for effects of group (sensitized, nonsensitized, salinetreated). Next, data from cells that could be definitively localized to either the accumbens core or the shell were analyzed for effects of group and location. Significant interactions were followed up with one-way ANOVAs to assess the effect of group at each anatomical location. Significant main effects were followed by independent $t$ tests. For cells that were recorded after acute administration of Meth, a subset of these baseline measures (membrane potential, up-state duration, up-state frequency, percentage of time in up state, and firing rate) were analyzed for the effect of time, using separate one-way ANOVAs for each group. For neurons that were recorded after PFC stimulation, the measures of response amplitude, latency, and decay were analyzed with a two-way mixed ANOVA for effects of group and membrane potential state (up vs down). Responses to VTA stimulations were analyzed by examining response amplitude, decay, and duration in a similar two-way mixed ANOVA. Finally, the response to combined stimulation of the PFC and VTA was analyzed by comparing PFC response amplitudes before and after VTA stimulation with separate dependent $t$ tests performed for each group. An $\alpha$ level of 0.05 was used for all analyses.

\section{Results}

\section{Locomotor activity}

Animals $(n=35)$ were tested for their locomotor response to an acute dose of Meth $(0.5 \mathrm{mg} / \mathrm{kg}$, i.p.) both before (baseline) and $13 \mathrm{~d}$ after (test) a regimen of five daily Meth injections (5.0 mg/kg, i.p.). Behavioral sensitization was assessed by calculating each animal's percentage change in Meth-elicited locomotor activity during the $2 \mathrm{~h}$ activity period, from the baseline to the test phase. A separate set of animals $(n=15)$ was given saline injections throughout each phase. Figure 1 illustrates the behavioral response of all animals during the $2 \mathrm{~h}$ activity period on the baseline and the test challenge days, after repeated Meth or saline administration. Animals that received repeated Meth were classified as either sensitized ( $\geq 50 \%$ change from baseline) or nonsensitized ( $\leq 25 \%$ change from baseline) after the test phase. Of a total of 194 animals that were treated with repeated Meth, a majority $(121 ; 62 \%)$ were thus classified as behaviorally sensitized, whereas 56 animals (29\%) were classified as nonsensitized and 17 (9\%) were not classified in either group because of a test phase response of between 25 and 50\% above baseline. From these 177 sensitized and nonsensitized Meth-treated animals, successful intracellular recordings were obtained from 35 rats. The proportion of behaviorally sensitized and nonsensitized animals was mostly preserved among these 35 animals, with 22 (63\%) classified as behaviorally sensitized and 13 (37\%) classified as nonsensitized.

Sensitized animals (Fig. 1a) exhibited a significant enhancement of Meth-elicited locomotor activity in the test phase compared with the baseline phase $\left(F_{(1,21)}=86.3 ; p<0.001\right)$ (Fig. 1d). Furthermore, in sensitized animals, the locomotor response to Meth took longer to dissipate in the test phase than in the baseline phase $\left(F_{(11,231)}=10.7 ; p<0.001\right)$. As predicted by their post hoc classification status, nonsensitized animals (Fig. $1 b$ ) exhibited no significant change in either overall Meth-elicited locomotor activity $\left(F_{(1,12)}=3.31 ; p=0.09\right)$ (Fig. $\left.1 d\right)$ or the pattern of locomotor activity across time $\left(F_{(11,132)}=1.69 ; p=0.12\right)$ from the baseline to the test phase. Saline-treated animals $(n=15)$ (Fig. 1c) did exhibit a higher level of locomotor activity during the $2 \mathrm{~h}$ period in the test phase than in the baseline phase $\left(F_{(1,14)}=36.5\right.$; $p<0.001)$, but this increase did not interact with time $\left(F_{(11,154)}=\right.$ $1.1 ; p=0.37)$. Activity levels during the $30 \mathrm{~min}$ habituation period (after saline injection) in the baseline and test phases differed according to sensitization status $\left(F_{(2,47)}=6.39 ; p=0.004\right)$. Specifically, Meth-treated animals subsequently classified as nonsensitized were more active than sensitized animals during the habituation period of the baseline phase (sensitized activity counts, $399 \pm 135$; nonsensitized, $498 \pm 134 ; t_{(33)}=2.12 ; p=0.042$ ), but not the test phase (sensitized, $420 \pm 117$; nonsensitized, $418 \pm$ $109 ; t_{(33)}=0.05 ; p=0.96$ ). This was attributable to a significant decrease in habituation period activity from the baseline phase to 
the test phase in nonsensitized animals only $\left(t_{(12)}=2.95 ; p=0.012\right)$.

Thus, repeated Meth administration enhanced the subsequent locomotor response to acute Meth in most animals but failed to do so in many others. We took advantage of this difference to examine physiological differences associated with these different behavioral outcomes although all animals received the same drug schedule.

\section{Baseline electrophysiological properties} of nucleus accumbens cells

Successful in vivo intracellular recordings were made from 23 cells in 22 sensitized Meth-treated animals, 15 cells in 13 nonsensitized Meth-treated animals, and 15 cells in 15 saline-treated animals. Accumbens neurons from animals in all three groups exhibited up and down membrane potential states (Fig. 2), as previously observed in accumbens cells from normal animals in vivo (O'Donnell and Grace, 1995; Goto and O'Donnell, 2001; Brady and O'Donnell, 2004). However, there was a significant difference among the three treatment groups in the time spent in the up state $\left(F_{(2,50)}=3.16 ; p=0.05\right)$, and a trend for significance in up-state duration $\left(F_{(2,50)}=2.92 ; p=0.06\right)$. Specifically, cells from nonsensitized animals spent a lower percentage of time in the up state $(35.6 \%)$ than cells from sensitized animals $(46.9 \%$; $\left.t_{(36)}=2.31 ; p=0.03\right)$, in part because of the tendency of cells from nonsensitized animals to have shorter up states (sensitized, $558 \pm 154 \mathrm{~ms}$; nonsensitized, $447 \pm 168 \mathrm{~ms} ; t_{(36)}=2.09 ; p=$ $0.04)$. These up states were also significantly shorter than in cells from saline-treated animals $\left(559 \pm 124 \mathrm{~ms} ; t_{(28)}=2.06 ; p=\right.$ $0.05)$, although the comparison of percentage of time spent in the up state $(44.3 \%)$ did not reach statistical significance $t_{(28)}=$ $1.58 ; p=0.12)$. No group differences were observed in the membrane potential of the down or up states, or the frequency of up states (all $p>0.27$ ). However, the minimum level of injected current required to evoke action potentials (rheobase) was altered, with accumbens neurons from nonsensitized animals $(n=8)$ showing an increased excitability (manifested as a lower rheobase value) compared with both sensitized animals $(n=17$; $\left.t_{(23)}=2.81 ; p=0.01\right)$ and saline-treated animals $\left(n=12 ; t_{(18)}=\right.$ $2.15 ; p=0.046)$.

Of the 53 neurons recorded, 41 could be definitively localized to either the accumbens core or shell region, based on calbindin immunoreactivity. Analysis of the electrophysiological properties of this subset of 41 cells (Table 1 ) revealed no group or regional differences in membrane potential (down or up state) or up-state frequency (all $p>0.57$ ). However, up-state duration among the different sensitization groups did depend on anatomical location. Duration of the up state differed according to sensitization group status in neurons from the accumbens shell $\left(F_{(2,11)}=11.24 ; p=0.002\right)$ (Fig. $\left.2 a, b\right)$ but not the core $\left(F_{(2,24)}=\right.$ $0.28 ; p=0.76$ ) (Fig. $2 c, d$ ). Specifically, as seen in the overall analysis, shell neurons from nonsensitized animals exhibited shorter up states than cells from either sensitized $\left(t_{(7)}=3.57 ; p=\right.$
C

Sensitized - core

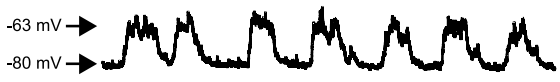

Non-sensitized - core

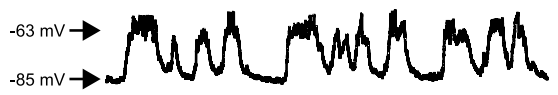

Saline - core

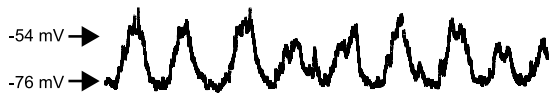

d
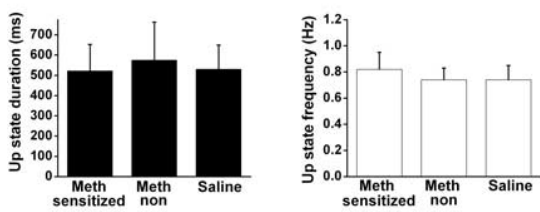

d

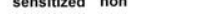

$$
\text { sensitized non }
$$

sensitzed non saline admin

Figure 2. Baseline electrophysiological properties in accumbens shell and core neurons after repeated Meth or saline admin-
istration. $\boldsymbol{a}$, Representative traces from shell neurons recorded in behaviorally sensitized, nonsensitized, and saline-treated animals. All cells exhibited up and down states, but up states were shortened in shell neurons from nonsensitized animals. $\boldsymbol{b}$, Summary of results (mean \pm SD) from all shell neurons showing significant reduction in up-state duration, but not frequency, in neurons from nonsensitized animals. c, Representative traces from core neurons recorded in behaviorally sensitized, nonsensitized, and saline-treated animals. All cells exhibited up and down states with very similar parameters. $\boldsymbol{d}$, Summary of results from changes in up-state duration or frequency. ${ }^{*} p<0.02$ (independent $t$ test). Error bars indicate SD.

$0.009)$ or saline-treated animals $\left(t_{(8)}=3.75 ; p=0.013\right)$. Thus, shell neurons may adapt to repeated Meth by changing elements that sustain up states in animals that do not exhibit behavioral sensitization.

\section{Response of accumbens cells to acute} methamphetamine administration

We also explored whether acute administration of Meth during the recording session differently affected physiological properties in animals from all three treatment groups. In nonsensitized Meth-treated animals, acute Meth administration $(0.5 \mathrm{mg} / \mathrm{kg}$, i.p.) abolished the spontaneous up- and down-state fluctuations and depolarized the membrane to a value close or even beyond the up state, in all five neurons tested (Fig. $3 b$ ). This effect was not seen after acute injection of saline $(n=2)$ (data not shown). The time course of the effect of acute Meth corresponds approximately to the peak of the locomotor response to the same dose of Meth during behavioral testing (Fig. 1). Approximately $20 \mathrm{~min}$ after Meth administration, the membrane potential of these neurons was $-63.6 \pm 4.8 \mathrm{mV}$, with no spontaneous oscillations. Although this value was significantly more depolarized than the up state of these neurons before acute drug treatment $(-72.4 \pm$ $\left.6.6 \mathrm{mV} ; F_{(3,12)}=3.97 ; p=0.041\right)$, spontaneous firing rates declined or stayed at zero in four of these five cells, and increased in only one cell (from 0 to $2.2 \mathrm{~Hz}$ ). Similarly, in saline-treated animals, three of four cells tested exhibited a loss of up- and downstate fluctuations after acute Meth administration (Fig. 3c). At 20 min after Meth injection, the resting membrane potential in these three neurons was not bimodal, but was relatively stable at 
Table 1. Electrophysiological properties of accumbens neurons

\begin{tabular}{|c|c|c|c|c|c|c|}
\hline & \multicolumn{3}{|c|}{ Nucleus accumbens shell } & \multicolumn{3}{|c|}{ Nucleus accumbens core } \\
\hline & Sensitized & Nonsensitized & Saline & Sensitized & Nonsensitized & Saline \\
\hline$n$ (cells/animals) & $4 / 4$ & $5 / 5$ & $5 / 5$ & $14 / 13$ & $5 / 4$ & $8 / 8$ \\
\hline \multicolumn{7}{|l|}{ Membrane potential (mV) } \\
\hline Down state & $-87.3 \pm 7.9$ & $-83.8 \pm 9.4$ & $-82.6 \pm 8.0$ & $-85.8 \pm 7.3$ & $-88.5 \pm 2.5$ & $-83.6 \pm 4.8$ \\
\hline Up state & $-71.8 \pm 10.4$ & $-73.1 \pm 6.7$ & $-67.5 \pm 6.1$ & $-69.1 \pm 7.2$ & $-70.8 \pm 6.1$ & $-69.0 \pm 8.7$ \\
\hline Time in up state (\%) & $58.2 \pm 9.4^{a}$ & $33.3 \pm 20.3^{a}$ & $50.3 \pm 9.5$ & $42.7 \pm 11.8$ & $41.7 \pm 13.9$ & $39.1 \pm 10.2$ \\
\hline Input resistance (M $\Omega$ ) & $53.8 \pm 16.9$ & $75.4 \pm 29.8$ & $83.0 \pm 40.4$ & $85.4 \pm 26.9$ & $104.7 \pm 28.0$ & $83.0 \pm 17.9$ \\
\hline Time constant $(\tau)$ (ms) & $5.9 \pm 1.7$ & $3.9 \pm 1.4$ & $5.2 \pm 0.9$ & $5.2 \pm 1.7$ & $5.2 \pm 1.8$ & $5.5 \pm 1.4$ \\
\hline Spike threshold (mV) & $-48.3 \pm 4.1$ & $-45.4 \pm 5.5$ & $-48.0 \pm 5.2$ & $-49.7 \pm 4.3^{b}$ & $-52.6 \pm 2.6^{b}$ & $-43.7 \pm 6.8^{b}$ \\
\hline Spike duration (ms) & $2.4 \pm 0.3^{c}$ & $1.8 \pm 0.2^{c}$ & $2.1 \pm 0.3$ & $2.2 \pm 0.4^{b}$ & $2.1 \pm 0.1^{b}$ & $2.9 \pm 0.9^{b}$ \\
\hline Rheobase $(\mathrm{nA})^{*}$ & $0.30 \pm 0.08$ & $0.23 \pm 0.06$ & $0.25 \pm 0.13$ & $0.30 \pm 0.05$ & $0.22 \pm 0.13$ & $0.36 \pm 0.11$ \\
\hline Firing rate $(\mathrm{Hz})$ & $\begin{array}{l}0.90 \pm 0.93 \\
(n=2 / 4)\end{array}$ & $\begin{array}{c}0.13 \pm 0 \\
(n=1 / 5)\end{array}$ & $\begin{array}{l}1.55 \pm 0 \\
(n=1 / 5)\end{array}$ & $\begin{array}{l}1.01 \pm 1.24 \\
(n=5 / 14)\end{array}$ & $\begin{array}{c}2.25 \pm 0.64 \\
(n=2 / 5)\end{array}$ & $\begin{array}{l}1.02 \pm 0.13 \\
(n=2 / 8)\end{array}$ \\
\hline
\end{tabular}

All values are mean \pm SD.

Independent $t$ test: ${ }^{a} p<0.06{ }^{b} p<0.05 ;{ }^{c} p<0.02$ versus saline.

*Main effect of group, $p<0.05$; independent $t$ test, nonsensitized versus sensitized $(p<0.01)$ or saline $(p<0.05)$.

\section{a}

Sensitized - baseline

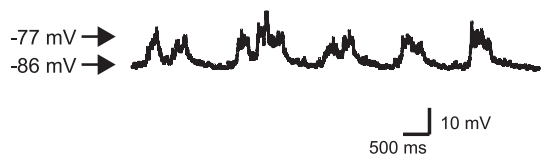

b

Non-sensitized - baseline

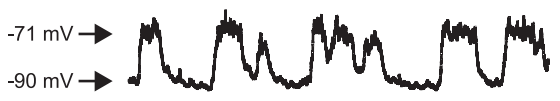

C

Saline - baseline

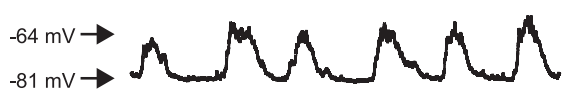

Sensitized - Meth challenge

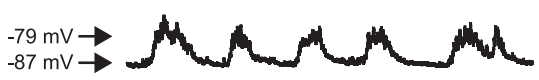

Non-sensitized - Meth challenge

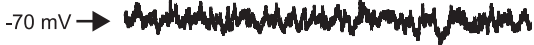

Saline - Meth challenge

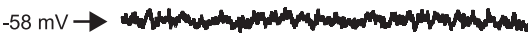

Figure 3. The effect of acute Meth challenge injection $(0.5 \mathrm{mg} / \mathrm{kg}$, i.p.) on up and down states in accumbens neurons depends on the behavioral response to repeated Meth administration. $\boldsymbol{a}$, Traces of a representative neuron from a behaviorally sensitized animal, before and $19 \mathrm{~min}$ after acute Meth administration. No changes in up- and down-state parameters were observed in the three of four cells (all in the accumbens core) that exhibited this type of response. $\boldsymbol{b}$, Representative neuron from a nonsensitized animal, before and $18 \mathrm{~min}$ after Meth injection. Note the loss of up and down states. This transition to a steady depolarized state was observed in five of five cells tested ( 3 in core, 1 in shell, 1 in rostral pole of the accumbens). $c$, Representative neuron from a saline-treated animal, before and 17 min after administration of Meth challenge. Up and down states were abolished in three (2 core; 1 shell) of four cells tested (the fourth cell was in the core).

$-53.9 \pm 6.8 \mathrm{mV}$, significantly more depolarized than baseline $\left(-65.1 \pm 9.6 \mathrm{mV} ; F_{(3,6)}=9.63 ; p=0.013\right)$. Spontaneous firing was reduced or remained at zero in all three of these cells. In contrast, accumbens neurons from sensitized Meth-treated animals continued to exhibit spontaneous up and down states in three of four cases after acute Meth administration (Fig. 3a), with the fourth cell displaying a loss of oscillations. In these three neurons from sensitized animals, the up- and down-state fluctuations persisted for at least $20 \mathrm{~min}$ postdrug. No changes were observed in the membrane potential of the up or down states, the duration or frequency of the up states, or spontaneous firing rates (all $p>0.15$ ). The proportion of neurons that continued to display up and down states after acute Meth was significantly higher in sensitized animals than in nonsensitized animals ( $p=0.047$; Fisher exact test). Thus, systemic administration of an acute "challenge" dose of Meth during the recording session had differential effects on accumbens neurons from sensitized, nonsensitized, and saline-treated animals.

\section{Response of accumbens cells to PFC and VTA afferent stimulation}

For all stimulations, there was an unequal distribution of neurons recorded in the accumbens core and shell, leading to very low $N$ in some cases; consequently, neurons were pooled across location for all subsequent analyses. Single-pulse stimulation of the PFC alone $(0.8-1.0 \mathrm{~mA} ; 0.5 \mathrm{~ms})$ elicited EPSPs in all 40 accumbens cells tested (Fig. 4a). There were no differences in the amplitude, latency, or decay of this synaptic response among neurons from sensitized $(n=16)$, nonsensitized $(n=9)$, or saline-treated $(n=15)$ animals (all $p>$ 0.57 ) (Fig. 4b). As previously observed in accumbens neurons from naive animals (Goto and O'Donnell, 2002; Brady and O'Donnell, 2004), EPSP amplitudes were larger $\left(F_{(1,26)}=32.1 ; p<0.001\right)$ and decay times were shorter $\left(F_{(1,26)}=30.08 ; p<\right.$ $0.001)$ when PFC stimulations occurred during accumbens down states than when the PFC was stimulated during PFC up states, but neither of these effects differed among cells from the three groups of animals in this experiment (amplitude, $F_{(2,26)}=$ $0.87, p=0.43$; decay, $\left.F_{(2,26)}=0.16, p=0.85\right)$. VTA stimulation in a pattern mimicking DA cell burst firing $(20 \mathrm{~Hz}$ train of five 1.0 $\mathrm{mA}, 0.5 \mathrm{~ms}$ pulses) produced a sustained depolarization in 37 of 38 accumbens neurons tested (Fig. $4 c$ ). The one cell that did not respond to VTA stimulation was from a Meth-treated, sensitized animal. The amplitude, decay, and duration of the VTA-evoked plateau depolarization were similar among neurons from sensitized Meth-treated $(n=16)$, nonsensitized Meth-treated $(n=9)$, and saline-treated $(n=12)$ animals (all $p>0.20)$ (Fig. $4 d)$. Thus, somewhat unexpectedly, no apparent differences in evoked re- 
a
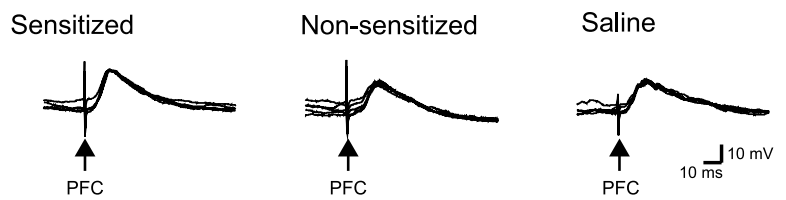

b
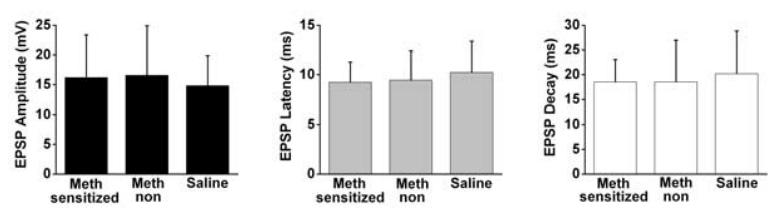

C

\section{d}

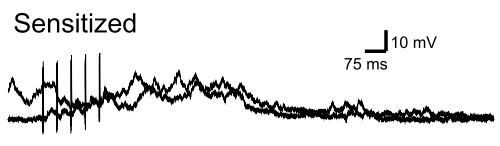

Non-sensitized
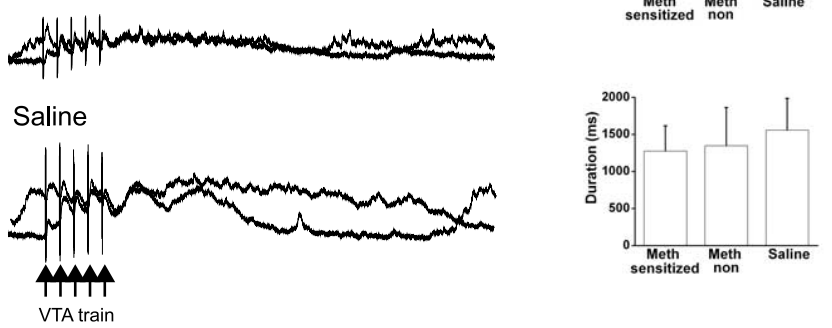

Figure 4. Responses to independent stimulation of the PFC or VTA are unaltered after repeated Meth or saline administration. $\boldsymbol{a}$, Single-pulse stimulation of the PFC elicited a similar EPSP in accumbens neurons from behaviorally sensitized ( $n=16)$, nonsensitized ( $n=9)$, and saline-treated ( $n=15$ ) animals. Traces are overlays of four to five sweeps per cell. $\boldsymbol{b}$, Summary of results (mean \pm SD) from all recorded neurons, illustrating no changes in the amplitude, onset latency, or decay time of the PFC-elicited response across the three groups. c, Train stimulation of the VTA provoked a long-lasting depolarization in neurons from behaviorally sensitized ( $n=16)$, nonsensitized $(n=9)$, and saline-treated ( $n=12$ ) animals. Traces are overlays of two sweeps per cell. $\boldsymbol{d}$, Summary of results from all recorded neurons, illustrating no changes in the amplitude or duration of the VTA-elicited plateau across the three groups. Error bars indicate SD.

sponses to either the PFC or the VTA were observed among experimental groups.

Although there were no group differences in the response to independent stimulation of the PFC or VTA, the effect of combined stimulation of these afferent pathways was altered in behaviorally sensitized animals. We showed previously that train stimulation of the VTA dampens PFC-elicited responses in accumbens neurons, an effect that was mediated by $\mathrm{D}_{2}$ dopamine receptors and was speculated to provide a mechanism to filter irrelevant information (Brady and O'Donnell, 2004). Here, this effect was reproduced in accumbens cells from nonsensitized animals $(n=4)$ and saline-treated animals $(n=7)$. In both groups, the amplitude of PFC-elicited EPSPs was diminished during VTA-evoked plateau depolarizations, compared with those elicited during a naturally occurring up state (nonsensitized, $t_{(3)}=$ 6.39, $p=0.008$; saline, $t_{(6)}=2.89, p=0.028$ ) (Fig. 5). However, the ability of VTA train stimulation to reduce the amplitude of PFC-elicited responses in accumbens neurons was abolished in behaviorally sensitized animals $\left(n=8 ; t_{(7)}=1.18 ; p=0.28\right)$ (Fig. $5)$, suggesting that mesolimbic filtering mechanisms may be dysfunctional in sensitized animals.

\section{Discussion}

The experiments reported here provide evidence for the disruption of multiple synaptic gating mechanisms in nucleus accumbens neurons after repeated Meth administration. The nature of these alterations depends crucially on whether animals exhibit behavioral sensitization in response to repeated drug treatment. On one hand, accumbens neurons from nonsensitized animals exhibit shorter up states compared with saline-treated and sensitized Meth-treated animals; however, the ability of VTA stimulation to reduce PFC-evoked EPSP amplitude and the ability of acute Meth to abolish up-down state oscillations are selectively lost in sensitized animals. These could represent adaptive and nonadaptive compensatory responses to repeated drug administration that may underlie the development of compulsive drug-seeking.

Altered accumbens activity in behaviorally sensitized animals After repeated Meth injections, a majority of animals exhibited enhanced locomotion after a subsequent challenge injection of Meth, which is a defining characteristic of behavioral sensitization. However, some animals displayed no changes in locomotor activity despite identical Meth experience, suggesting that there are individual differences in the behavioral response to psychostimulants. Similar variations have previously been reported by others (Hooks et al., 1991; Pierce et al., 1996; Churchill et al., 1999; Sabeti et al., 2003; Kozell and Meshul, 2004), indicating that animals, like humans (Volkow et al., 2004), differ in their vulnerability to drugs of abuse. In addition, nonsensitized animals were more active than sensitized animals during the habituation period at baseline (i.e., during the initial exposure to a novel environment and a saline injection). There was no difference between groups during the habituation period of the test phase, in general agreement with published reports of high and low responders to novelty (Hooks et al., 1991; Pierre and Vezina, 1997). The finding that animals with a high response to novelty/saline failed to sensitize to repeated Meth injections concurs with some previous reports (Piazza et al., 1989) but not others (Hooks et al., 1991). These results suggest the presence of group differences in responsiveness to novelty and/or stress both before and after repeated Meth injections. However, the available data do not allow for additional analysis of these findings, and a full discussion of the behavioral characteristics of sensitized and nonsensitized animals is beyond the scope of this report (Hooks et al., 1991; Sabeti et al., 2003). Nevertheless, the behavioral differences between sensitized and nonsensitized animals appear to be functionally and neurobiologically relevant, because various neuroadaptations in the nucleus accumbens or VTA are reported to develop selectively in behaviorally sensitized animals (Pierce et al., 1996; Churchill et al., 1999; Sabeti et al., 2003).

Accumbens neurons from sensitized animals were surprisingly unaffected on many baseline measures. Instead, we found the most pronounced group differences in spontaneous activity in accumbens neurons from nonsensitized animals, which spent less time in the up state. Because the up state represents an active phase during which the response of a neuron to excitatory inputs (especially from the PFC) is facilitated (O'Donnell and Grace, 1995), the reduction in up-state duration implies that these neurons will be less responsive to afferent inputs, potentially causing an overall reduction in the output of accumbens neurons from nonsensitized animals. These baseline changes were observed mainly in the nucleus accumbens shell, in accordance with some previous findings in sensitized animals (Pierce and Kalivas, 1995; Filip and Siwanowicz, 2001), although other evidence supports a 
a
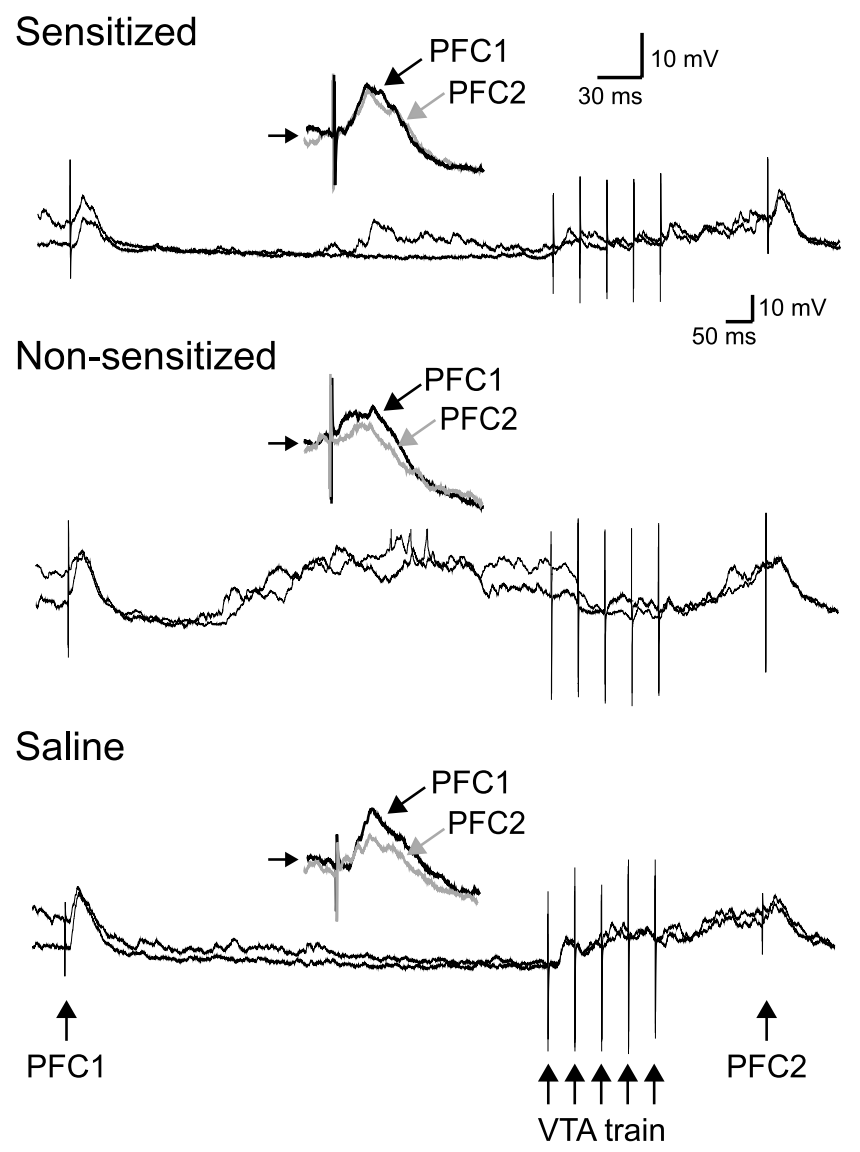

b

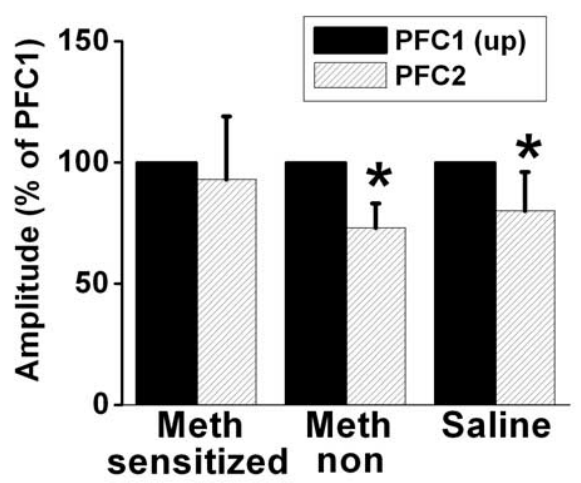

Figure 5. The ability of VTA stimulation to attenuate PFC-elicited responses in accumbens neurons is selectively disrupted in behaviorally sensitized animals. $\boldsymbol{a}$, The synaptic response to PFC stimulation was attenuated by VTA stimulation in accumbens cells from nonsensitized ( $n=$ 4 ) and saline-treated $(n=7)$ animals. In these neurons, the amplitude of EPSPs was lower when the PFC was stimulated after VTA train stimulation (PFC2) than when it was stimulated at the same intensity at baseline (PFC1). However, in accumbens cells from behaviorally sensitized animals $(n=8)$ ), PFC-elicited EPSPs were not altered by previous VTA stimulation (PFC2 amplitude was equal to PFC1 amplitude). Two sweeps from each cell are overlaid to show the response to PFC1 stimulation during a spontaneous up or down state. Insets, Detail of the EPSPs evoked by PFC stimulation at a depolarized membrane potential (small arrow), occurring during either a spontaneous up state (PFC1, black trace) or a VTA-evoked plateau (PFC2, grayed trace). The reduction in PFC2 amplitude can be seen in nonsensitized and saline-treated, but not sensitized, animals. $\boldsymbol{b}$, Summary of normalized results from all recorded neurons (mean \pm SD), showing a significant reduction of PFC response amplitude after VTA stimulation (compared with spontaneous up states) in nonsensitized and saline-treated animals. No such reduction was observed in accumbens cells from sensitized animals. ${ }^{*} p<0.03$ (repeated $t$ tests). Error bars indicate SD. primary role of the accumbens core in psychostimulant sensitization (Cadoni et al., 2000; Hedou et al., 2002).

The lack of changes in accumbens neurons in response to afferent stimulation of the PFC or VTA was also unexpected, in light of the observed reduction in up-state duration as well as previous reports showing that repeated psychostimulant administration can alter responses to glutamatergic or dopaminergic innervation (Wolf et al., 1994; White et al., 1995), including prefrontal inputs (Thomas et al., 2001). In the current study, we directly activated afferent pathways in vivo, whereas previous results have used exogenously applied drugs, or afferent stimulation in vitro. However, even in vivo stimulation fails to wholly reproduce endogenous patterns of afferent activity in the awake, behaving animal. Thus, the negative results reported here do not rule out the possibility of other alterations in these pathways; for example, changes in somatic excitability in the PFC (Peterson et al., 2000; Trantham et al., 2002) or VTA (Wolf et al., 1993; White et al., 1995) would presumably be translated into changes in the strength and pattern of accumbens inputs.

Stimulation of the VTA dampened the subsequent accumbens response to glutamatergic PFC inputs (as previously observed in naive animals) (Brady and O'Donnell, 2004) in saline-treated and nonsensitized, but not behaviorally sensitized animals. Because this inhibition is dependent on $\mathrm{D}_{2}$ receptor stimulation (O'Donnell and Grace, 1994; Brady and O'Donnell, 2004), its absence in sensitized animals indirectly suggests a reduction in $\mathrm{D}_{2}$ receptor activation, an effect linked clinically to both chronic drug use and a potential vulnerability to drug abuse (Volkow et al., 2004). In contrast to these results, others have reported an enhancement of the inhibitory effects of dopamine on accumbens neurons (Higashi et al., 1989; Wolf et al., 1994; Beurrier and Malenka, 2002). Disparities between these findings and our results may derive from methodological differences in recording conditions and stimulation parameters, as well as differences in the psychostimulant agent used (Vanderschuren and Kalivas, 2000).

Our results suggest that overall excitatory drive to accumbens neurons may not be globally reduced (White et al., 1995; Zhang et al., 1998; Thomas et al., 2001; Beurrier and Malenka, 2002), but the balance among excitatory inputs may be disrupted. Dopamine is thought to play a role in achieving this balance by mediating the gating and selection of appropriate inputs for processing by accumbens neurons (Mogenson et al., 1988; Floresco et al., 2001; O'Donnell, 2003). Here, the disruption of one such gating function in sensitized animals implies that arriving inputs from the PFC are not subject to normal filtering by concurrent accumbens dopamine transmission, which may lead to inappropriate processing of irrelevant information. In addicted individuals, such disruptions of dopamine function could lead to the assignment of a pathological level of salience to drug-associated cues, at the expense of appropriate processing of cues associated with natural events and reinforcers (Volkow et al., 2004).

\section{Accumbens neuroadaptations as putative compensatory mechanisms}

Repeated stimulant administration may cause neuroadaptations in accumbens circuits. The shorter up states and increased excitability observed in cells from nonsensitized animals could represent compensatory mechanisms of tolerance that develop in response to repeated Meth administration. It may be further speculated that such tolerance mechanisms function adaptively to allow accumbens neurons to continue normal functioning despite repeated exposure to massive increases in accumbens dopa- 
mine transmission, and that these adaptations may prevent the manifestation of behavioral sensitization. Alternatively, these mechanisms could reflect preexisting individual differences (Hooks et al., 1991; Marinelli and White, 2000) that may confer variable levels of vulnerability on animals, thus contributing to whether or not animals will exhibit behavioral sensitization after repeated drug exposure. These mechanisms could then allow nonsensitized accumbens neurons to function normally in response to complex afferent stimulation, as evidenced by the preservation of dopaminergic attenuation of PFC inputs in the current study, and previous reports suggesting normal glutamatergic transmission in drug-exposed, nonsensitized animals (Pierce et al., 1996; Churchill et al., 1999). Such compensatory cellular mechanisms are presumed to be absent in behaviorally sensitized animals, and/or neurons from these animals could develop aberrant compensatory mechanisms that manifest as normalappearing basal electrophysiological properties, but interfere with the response to complex synaptic inputs. The reported increase in the number of spines, which are the primary targets of both dopamine and glutamate inputs, on accumbens neurons after repeated psychostimulant exposure may be one mechanism by which the precise functional arrangement of synaptic inputs is disrupted (Robinson and Kolb, 2004).

The hypothesized development of maladaptive neuroadaptations in accumbens neurons from sensitized animals is also supported by the effects of an acute Meth challenge. In saline-treated animals, acute Meth abolished the spontaneous occurrence of up and down states. This was characterized by a depolarization beyond the up-state value and is consistent with the strong depolarization by dopamine observed in accumbens neurons in slices, discussed previously as possibly mediated by reduced potassium conductances (O'Donnell and Grace, 1996). Because these animals were previously drug-naive, this effect is assumed to represent a "normal" response to acute psychostimulant exposure. However, in behaviorally sensitized animals, acute Meth failed to disrupt membrane potential oscillations. The selective observation of this effect in accumbens neurons only from sensitized animals suggests that it has functional relevance for the neurobiological substrates of behavioral sensitization. In a similar vein, acute amphetamine loses its normal ability to block certain forms of long-term potentiation at accumbens synapses in vitro after repeated amphetamine treatment in vivo (Li and Kauer, 2004). Together with the current results, these findings suggest that, after repeated drug administration, there is a breakdown in the regulatory mechanisms that normally limit accumbens information processing in the presence of acute exposure to psychostimulants, possibly via a reduction in the dopaminergic modulation of glutamate transmission (Li and Kauer, 2004). Gating and plasticity mechanisms are allowed to continue despite massive drug-induced increases in accumbens dopamine transmission, perhaps leading to aberrant processing of irrelevant environmental cues and the inappropriate assignment of incentive salience to drug-related stimuli (Robinson and Berridge, 1993; Volkow et al., 2004). Thus, neuroadaptations that allow accumbens information processing to continue under the acute influence of psychostimulant drugs would represent maladaptive compensatory mechanisms that develop in response to chronic drug use.

\section{Conclusions}

Here, we demonstrated changes in the electrophysiological properties of accumbens neurons that suggest both the development of adaptive mechanisms in animals that are resistant to the sen- sitizing effects of methamphetamine, and the occurrence of maladaptive compensatory mechanisms in drug-vulnerable animals. These maladaptive mechanisms in behaviorally sensitized animals result in the disrupted integration of dopamine and glutamate inputs to the accumbens, and the failure of acute drug exposure to alter synaptic gating mechanisms in accumbens neurons. After repeated drug use, such neuroadaptations may compromise an organism's ability to select the appropriate behavioral response in situations that require the precise integration of multiple inputs to the nucleus accumbens. These findings support the idea that repeated exposure to drugs of abuse usurps and distorts the cellular mechanisms that normally govern learning and plasticity in forebrain circuits (Wolf et al., 2004), leading to compulsive drug use despite negative consequences (Hyman et al., 2001).

\section{References}

Beurrier C, Malenka RC (2002) Enhanced inhibition of synaptic transmission by dopamine in the nucleus accumbens during behavioral sensitization to cocaine. J Neurosci 22:5817-5822.

Brady AM, O'Donnell P (2004) Dopaminergic modulation of prefrontal cortical input to nucleus accumbens neurons in vivo. J Neurosci 24:1040-1049.

Cadoni C, Solinas M, Di Chiara G (2000) Psychostimulant sensitization: differential changes in accumbal shell and core dopamine. Eur J Pharmacol 388:69-76.

Cador M, Bjijou Y, Stinus L (1995) Evidence of a complete independence of the neurobiological substrates for the induction and expression of behavioral sensitization to amphetamine. Neuroscience 65:385-395.

Cador M, Bjijou Y, Cailhol S, Stinus L (1999) D-Amphetamine-induced behavioral sensitization: implication of a glutamatergic medial prefrontal cortex-ventral tegmental area innervation. Neuroscience 94:705-721.

Churchill L, Swanson CJ, Urbina M, Kalivas PW (1999) Repeated cocaine alters glutamate receptor subunit levels in the nucleus accumbens and ventral tegmental area of rats that develop behavioral sensitization. J Neurochem 72:2397-2403.

Deminiere JM, Piazza PV, Le Moal M, Simon H (1989) Experimental approach to individual vulnerability to psychostimulant addiction. Neurosci Biobehav Rev 13:141-147.

De Vries TJ, Schoffelmeer AN, Binnekade R, Mulder AH, Vanderschuren LJ (1998) Drug-induced reinstatement of heroin- and cocaine-seeking behaviour following long-term extinction is associated with expression of behavioural sensitization. Eur J Neurosci 10:3565-3571.

Filip M, Siwanowicz J (2001) Implication of the nucleus accumbens shell, but not core, in the acute and sensitizing effects of cocaine in rats. Pol J Pharmacol 53:459-466.

Floresco SB, Blaha CD, Yang CR, Phillips AG (2001) Modulation of hippocampal and amygdalar-evoked activity of nucleus accumbens neurons by dopamine: cellular mechanisms of input selection. J Neurosci 21:2851-2860.

Goto Y, O’Donnell P (2001) Network synchrony in the nucleus accumbens in vivo. J Neurosci 21:4498-4504.

Goto Y, O’Donnell P (2002) Timing-dependent limbic-motor synaptic integration in the nucleus accumbens. Proc Natl Acad Sci USA 99:13189-13193.

Hedou G, Jongen-Relo AL, Murphy CA, Heidbreder CA, Feldon J (2002) Sensitized Fos expression in subterritories of the rat medial prefrontal cortex and nucleus accumbens following amphetamine sensitization as revealed by stereology. Brain Res 950:165-179.

Higashi H, Inanaga K, Nishi S, Uchimura N (1989) Enhancement of dopamine actions on rat nucleus accumbens neurones in vitro after methamphetamine pre-treatment. J Physiol (Lond) 408:587-603.

Hooks MS, Jones GH, Smith AD, Neill DB, Justice Jr JB (1991) Individual differences in locomotor activity and sensitization. Pharmacol Biochem Behav 38:467-470.

Hyman SE, Hyman SE, Malenka RC (2001) Addiction and the brain: the neurobiology of compulsion and its persistence. Nat Rev Neurosci 2:695-703.

Kauer JA (2004) Learning mechanisms in addiction: synaptic plasticity in 
the ventral tegmental area as a result of exposure to drugs of abuse. Annu Rev Physiol 66:447-475.

Kozell LB, Meshul CK (2004) Nerve terminal glutamate immunoreactivity in the rat nucleus accumbens and ventral tegmental area after a short withdrawal from cocaine. Synapse 51:224-232.

LiY, Kauer JA (2004) Repeated exposure to amphetamine disrupts dopaminergic modulation of excitatory synaptic plasticity and neurotransmission in nucleus accumbens. Synapse 51:1-10.

Lorrain DS, Arnold GM, Vezina P (2000) Previous exposure to amphetamine increases incentive to obtain the drug: long-lasting effects revealed by the progressive ratio schedule. Behav Brain Res 107:9-19.

Marinelli M, White FJ (2000) Enhanced vulnerability to cocaine selfadministration is associated with elevated impulse activity of midbrain dopamine neurons. J Neurosci 20:8876-8885.

Mendrek A, Blaha CD, Phillips AG (1998) Pre-exposure of rats to amphetamine sensitizes self-administration of this drug under a progressive ratio schedule. Psychopharmacology (Berl) 135:416-422.

Mogenson GJ, Yang CR, Yim CY (1988) Influence of dopamine on limbic inputs to the nucleus accumbens. Ann NY Acad Sci 537:86-100.

O’Donnell P (1999) Ensemble coding in the nucleus accumbens. Psychobiology 27:187-197.

O'Donnell P (2003) Dopamine gating of forebrain neural ensembles. Eur J Neurosci 17:429-435.

O’Donnell P, Grace AA (1994) Tonic D2-mediated attenuation of cortical excitation in nucleus accumbens neurons recorded in vitro. Brain Res 634:105-112.

O’Donnell P, Grace AA (1995) Synaptic interactions among excitatory afferents to nucleus accumbens neurons: hippocampal gating of prefrontal cortical input. J Neurosci 15:3622-3639.

O’Donnell P, Grace AA (1996) Dopaminergic reduction of excitability in nucleus accumbens neurons recorded in vitro. Neuropsychopharmacology 15:87-97.

Paulson PE, Robinson TE (1995) Amphetamine-induced time-dependent sensitization of dopamine neurotransmission in the dorsal and ventral striatum: a microdialysis study in behaving rats. Synapse 19:56-65.

Paulson PE, Camp DM, Robinson TE (1991) Time course of transient behavioral depression and persistent behavioral sensitization in relation to regional brain monoamine concentrations during amphetamine withdrawal in rats. Psychopharmacology (Berl) 103:480-492.

Peterson JD, Wolf ME, White FJ (2000) Altered responsiveness of medial prefrontal cortex neurons to glutamate and dopamine after withdrawal from repeated amphetamine treatment. Synapse 36:342-344.

Piazza PV, Deminiere JM, Le Moal M, Simon H (1989) Factors that predict individual vulnerability to amphetamine self-administration. Science 245:1511-1513.

Pierce RC, Kalivas PW (1995) Amphetamine produces sensitized increases in locomotion and extracellular dopamine preferentially in the nucleus accumbens shell of rats administered repeated cocaine. J Pharmacol Exp Ther 275:1019-1029.

Pierce RC, Kalivas PW (1997) A circuitry model of the expression of behavioral sensitization to amphetamine-like psychostimulants. Brain Res Brain Res Rev 25:192-216.

Pierce RC, Bell K, Duffy P, Kalivas PW (1996) Repeated cocaine augments excitatory amino acid transmission in the nucleus accumbens only in rats having developed behavioral sensitization. J Neurosci 16:1550-1560.

Pierce RC, Reeder DC, Hicks J, Morgan ZR, Kalivas PW (1998) Ibotenic acid lesions of the dorsal prefrontal cortex disrupt the expression of behavioral sensitization to cocaine. Neuroscience 82:1103-1114.

Pierre PJ, Vezina P (1997) Predisposition to self-administer amphetamine: the contribution of response to novelty and prior exposure to the drug. Psychopharmacology (Berl) 129:277-284.

Robinson TE, Berridge KC (1993) The neural basis of drug craving: an incentive-sensitization theory of addiction. Brain Res Brain Res Rev 18:247-291.

Robinson TE, Kolb B (2004) Structural plasticity associated with exposure to drugs of abuse. Neuropharmacology 47 [Suppl 1]:33-46.

Sabeti J, Gerhardt GA, Zahniser NR (2003) Individual differences in cocaine-induced locomotor sensitization in low and high cocaine locomotor-responding rats are associated with differential inhibition of dopamine clearance in nucleus accumbens. J Pharmacol Exp Ther 305:180-190.

Szumlinski KK, Balogun MY, Maisonneuve IM, Glick SD (2000) Interactions between iboga agents and methamphetamine sensitization: studies of locomotion and stereotypy in rats. Psychopharmacology (Berl) 151:234-241.

Thomas MJ, Beurrier C, Bonci A, Malenka RC (2001) Long-term depression in the nucleus accumbens: a neural correlate of behavioral sensitization to cocaine. Nat Neurosci 4:1217-1223.

Trantham H, Szumlinski KK, McFarland K, Kalivas PW, Lavin A (2002) Repeated cocaine administration alters the electrophysiological properties of prefrontal cortical neurons. Neuroscience 113:749-753.

Vanderschuren LJ, Kalivas PW (2000) Alterations in dopaminergic and glutamatergic transmission in the induction and expression of behavioral sensitization: a critical review of preclinical studies. Psychopharmacology (Berl) 151:99-120.

Volkow ND, Fowler JS, Wang GJ (2004) The addicted human brain viewed in the light of imaging studies: brain circuits and treatment strategies. Neuropharmacology 47 [Suppl 1]:3-13.

White FJ, Hu XT, Zhang XF, Wolf ME (1995) Repeated administration of cocaine or amphetamine alters neuronal responses to glutamate in the mesoaccumbens dopamine system. J Pharmacol Exp Ther 273:445-454.

Wolf ME, White FJ, Nassar R, Brooderson RJ, Khansa MR (1993) Differential development of autoreceptor subsensitivity and enhanced dopamine release during amphetamine sensitization. J Pharmacol Exp Ther 264:249-255.

Wolf ME, White FJ, Hu XT (1994) MK-801 prevents alterations in the mesoaccumbens dopamine system associated with behavioral sensitization to amphetamine. J Neurosci 14:1735-1745.

Wolf ME, Dahlin SL, Hu XT, Xue CJ, White K (1995) Effects of lesions of prefrontal cortex, amygdala, or fornix on behavioral sensitization to amphetamine: comparison with $N$-methyl-D-aspartate antagonists. Neuroscience 69:417-439.

Wolf ME, Sun X, Mangiavacchi S, Chao SZ (2004) Psychomotor stimulants and neuronal plasticity. Neuropharmacology 47 [Suppl 1]:61-79.

Zhang XF, Hu XT, White FJ (1998) Whole-cell plasticity in cocaine withdrawal: reduced sodium currents in nucleus accumbens neurons. J Neurosci 18:488-498. 